\title{
The first shoots of a modern morphometrics approach to the origins of agriculture
}

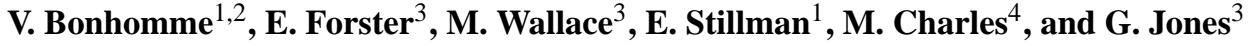 \\ ${ }^{1}$ School of Mathematics and Statistics, University of Sheffield, Hicks Building, \\ Hounsfield Road, Sheffield S3 7RH, UK \\ ${ }^{2}$ UMR 5554 Institut des Sciences de l'Evolution, équipe Dynamique de la biodiversité, \\ anthropo-écologie, Université de Montpellier, CNRS, IRD, EPHE Place Eugène Bataillon, \\ 34095 Montpellier, CEDEX 05, France \\ ${ }^{3}$ Department of Archaeology, University of Sheffield, Northgate House, West Street, Sheffield S1 4ET, UK \\ ${ }^{4}$ Institute of Archaeology, 36 Beaumont St, Oxford, OX1 2PG, UK
}

Correspondence to: V. Bonhomme (v.bonhomme@sheffield.ac.uk)

Received: 12 October 2015 - Revised: 4 December 2015 - Accepted: 9 December 2015 - Published: 18 January 2016

\section{Introduction}

The transition from a mobile hunter-gatherer lifestyle to one of settled agriculture is arguably the most fundamental change in the development of human society (Lev-Yadun et al., 2000). The establishment of agricultural economies, emerging initially in the Fertile Crescent of the Near East (Nesbitt, 2002), required the domestication of crops; ancient plant remains recovered from early farming sites provide direct evidence for this process of domestication. Archaeobotanical remains are typically preserved through charring (partial to complete carbonisation through exposure to heat) and recovered during archaeological excavation (Charles et al., 2015). Seeds of the same species, recovered from different sites and periods, can sometimes be seen to exhibit morphological differences, which may have arisen owing to variations in cultivation practices, climate, soils and altitude, etc. To explore these possibilities, morphological variation in seeds of wheat and barley between archaeological sites was recorded and mapped both in time and space. Results presented here suggest that modern morphometric approaches may help to test some long-debated hypotheses and pave the way for new insights into the evolutionary origins of agriculture in western Asia.

\section{Material and methods}

Grains of the "primitive" glume wheats einkorn (Triticum monococcum) and emmer (T. dicoccum), domesticated barley (Hordeum vulgare) and their wild progenitors ( $T$. boeoticum, $T$. dicoccoides and $H$. spontaneum respectively), dating from ca. 13000 to 9000 years before present, originating mainly from the Fertile Crescent region and now archived in various European institutions, were sampled. Three orthogonal views were photographed and grain outlines were converted into $x-y$ coordinates. The three views of each grain were processed independently and later combined for analysis. Traditional measurements were obtained directly (length, breadth, thickness) or estimated (ellipsoid volume) based on these outlines. Outlines were then centred, scaled and aligned, and elliptical Fourier transforms were calculated (Kuhl and Giardina, 1982). The number of harmonics was chosen to encompass $99 \%$ of the harmonic power for each view. Elliptical Fourier transforms turned the grain shapes into quantitative variables that were further treated using classical multivariate approaches, mainly ordination and classification. All calculations and analyses were accomplished using the $\mathrm{R}$ environment ( $\mathrm{R}$ Development Core Team, 2015) and the modern morphometrics package Momocs (Bonhomme, 2015; Bonhomme et al., 2014). Linear discriminant analyses were performed with the MASS package (Venables and Ripley, 2015).

\section{Results and discussion}

A total of 1378 grains were photographed in three orthogonal views, with 554 being of the genus Hordeum and 824 from Triticum. Grains were sampled from 14 prehistoric archaeological sites across southwest Asia (Fig. 1a). This, to our 


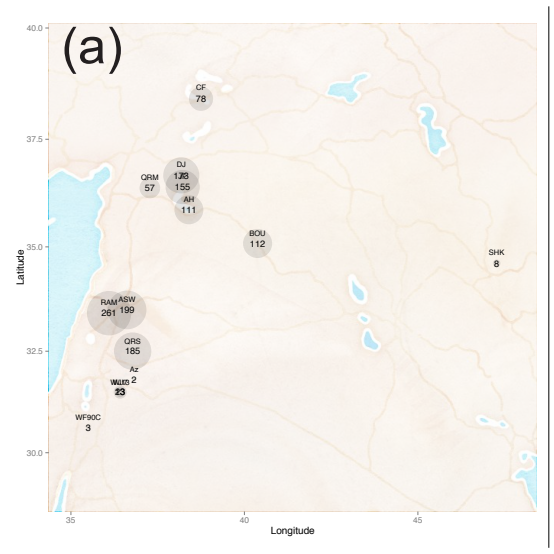

(b)

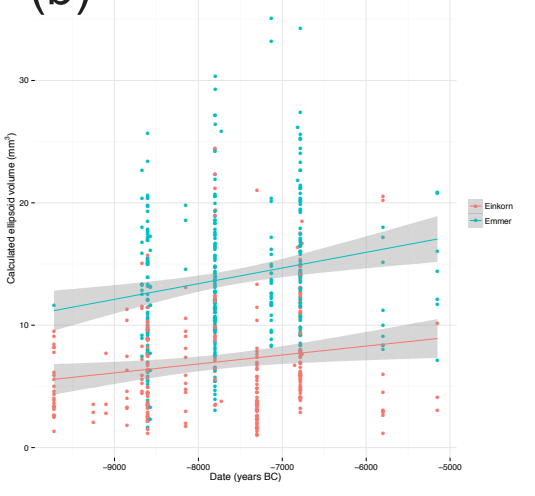

(c)

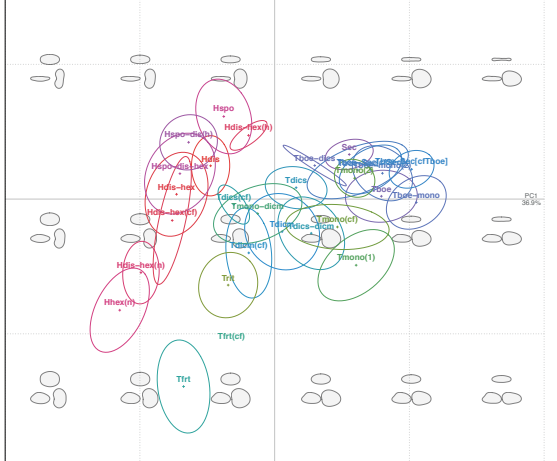

Figure 1. (a) Map of the archaeological sites along with their sample sizes. (b) The increasing trend for the grain volume observed in emmer and einkorn grains. The regression indicates a $50 \%$ increase in the grain volume over 5000 years. (c) The first two axes of the principal component analysis ( $\sim 70 \%$ of the total variance) along with confidence ellipses labelled with taxon names and, in the background, a morphospace of the reconstructed shapes with the three views arranged as dorsal above, lateral left and polar right. The red ensemble on the left gathers all Hordeum taxa; the blue/green ellipses are all Triticum taxa.

knowledge, is the largest photographic database of ancient grains ever collected. Increase in grain size has previously been associated with domestication of cereals (e.g. Fuller et al., 2011), and this trend can be seen clearly in our data set. For instance, for einkorn and emmer grains there is a $50 \%$ increase in volume between 10000 and $5000 \mathrm{BC}$ when considering all sites together (Fig. 1b).

Principal component analysis on the whole data set (Fig. 1c) illustrates the difference between Hordeum and Triticum taxa. Cross-validation (leave-one-out) at the genus level with a linear discriminant analysis suggests this can be feasible with a high confidence level in the classification (95 and $98 \%$ of correct attribution for Hordeum and Triticum grains, respectively). Whilst an automated classification system remains some way off, such analyses may prove a valuable tool for aiding the identification of crop remains by archaeobotanists. Although it is unlikely that this would be used for the most commonly identified taxa, morphometric analysis may help to separate types that are difficult to distinguish by eye, such as wild and domesticated forms of a species or taxa that are traditionally grouped as indistinguishable (e.g. wild einkorn (Triticum boeoticum) and rye (Secale spp.)). For instance, $86 \%$ of wild and $75 \%$ of domesticated einkorn (Triticum boeoticum and T. monococcum) grains are correctly classified when species information is removed.

To conclude, these first results of a modern morphometric approach on the largest photographic database of ancient grains ever collected are promising, and they pave the way for exploration of changes in grain size and shape through time and across geographic regions.

Acknowledgements. This research is funded by the European Research Council project "Evolutionary origins of agriculture" PI: Glynis Jones, grant number 269030. This is publication
ISEM 2015-254.

Edited by: D. Montesinos

Reviewed by: two anonymous referees

\section{References}

Bonhomme, V.: Momocs' repository, available at: https://github. com/vbonhomme/Momocs/, last access: 21 December 2015.

Bonhomme, V., Picq, S., Gaucherel, C., and Claude, J.: Momocs: Outline Analysis Using R, J. Stat. Softw., 56, doi:10.18637/jss.v056.i13, 2014.

Charles, M., Forster, E., Wallace, M., and Jones, G.: "Nor ever lightning char thy grain": establishing archaeologically relevant charring conditions and their effect on glume wheat grain morphology, STAR, 1, STAR20152054892315Y.0000000008, doi:10.1179/2054892315Y.0000000008, 2015.

Fuller, D. Q., Asouti, E., and Purugganan, M. D.: Cultivation as slow evolutionary entanglement: comparative data on rate and sequence of domestication, Veg. Hist. Archaeobot., 21, 131-145, 2011.

Kuhl, F. F. P. and Giardina, C. C. R.: Elliptic Fourier features of a closed contour, Comput. Vision Graph., 18, 236-258, 1982.

Lev-Yadun, S., Gopher, A., and Abbo, S.: The cradle of agriculture, Science, 288, 1602-1603, 2000.

Nesbitt, M.: When and where did domesticated cereals first occur in southwest Asia?, in: The dawn of farming in the Near EastCappers, Berlin: ex oriente, edited by: R. T. J. Cappers and Bottema, S., 113-132, 2002.

R Development Core Team: R: A Language and Environment for Statistical Computing, R Foundation for Statistical Computing, Vienna, Austria, 2015.

Venables, W. N. and Ripley, B. D.: Modern Applied Statistics with S, 4th Edition, Springer, New York, USA, ISBN 0-387-95457-0, 2002. 